\title{
MÉTODO HÍBRIDO DE MIGRAC̄̃O PRÉ-EMPILHAMENTO EM PROFUNDIDADE NO DOMÍNIO DA FREQÜÊECIA EM DUAS ETAPAS COM INTERPOLAÇÃO
}

\author{
Gary C. Aldunate ${ }^{1}$ e Reynam C. Pestana ${ }^{2}$ \\ Recebido em 28 novembro, 2005 / Aceito em 6 março, 2006 \\ Received on November 28, 2005 / Accepted on March 6, 2006
}

\begin{abstract}
This paper shows the application of Phase Shift Plus Interpolation Split-Step (PSPI-SS) wavefield extrapolation operator coupled with the excitationtime imaging condition to perform prestack seismic depth migration of common shot profiles. The migration methods which are characterized by the use of different propagators for the source and receiver wavefields are called hybrid migration methods. Normally, the source wavefield is determined by the computation of the traveltimes from the solution of the eikonal equation. Here the traveltimes were calculated based on a finite difference solution of the eikonal equation (TGRID), a simple geometrical approach (FAST) and also by the paraxial ray tracing method (PARAX). For the extrapolation of the receiver data we applied the PSPI Split-Step operator, that is derived from the conventional Split-Step wave equation method using multiple references velocity. As the source traveltime computation is based on the ray assintotic theory the velocity field needs to be smoothed and the migration results obtained with the Kirchhof migration method, that is a very fast migration procedure, was used to choose the best level of smoothing of the velocity field. In this work the synthetic Marmousi data and a real dataset from Gulf of Mexico were used to test the migration methods proposed here. The migration results with the hybrid methods shown better images in quality then the kirchhoff's results and comparable to those obtained with the full PSPI-SS migration method, but a low computation cost.
\end{abstract}

Keywords: Hybrid migration, Prestack migration, Split-Step Phase Shift Plus Interpolation.

RESUMO. Neste trabalho apresentamos a aplicação do operador de extrapolação em profundidade em duas etapas com interpolação (PSPI-SS), operador que faz uso da correção Split-Step em cada passo de extrapolação, conjuntamente com a condição de imagem de tempo de excitação, para realizar a migração pré-empilhamento em profundidade de dados sísmicos ordenados em famílias de tiro comum. Os métodos de migração pré-empilhamento que realizam a extrapolação dos dados ao longo dos receptores e a continuação para baixo das fontes sísmicas com operadores diferentes, são chamados de métodos híbridos de migração. Nesses métodos o campo de onda de cada fonte sísmica é determinado através do cálculo dos tempos de chegada da fonte a partir da aproximação de alta freqüência. Aqui utilizamos apenas os tempos das primeiras chegadas, que são calculados a partir da solução da equação iconal por diferença finitas (TGRID), por um método puramente geométrico (FAST) e também pelo método de traçamento de raios paraxiais (PARAX). Na implementação proposta, o campo de velocidades usado para o cálculo dos tempos de chegada precisa ser suavizado e o seu grau de suavização é determinado a partir dos resultados apresentados pela migração convencional Kirchhoff. A extrapolação dos dados dos receptores é feita usando-se o campo de velocidades original sem nenhuma suavização, através do operador PSPI-SS. Para testar os novos métodos de migração usamos os dados sintéticos Marmousi e também um conjunto de dados reais do Golfo do México. Os resultados das migrações com os métodos híbridos apresentaram imagens de boa qualidade, mostrando-se superiores a obtida pela migração Kirchhoff e comparáveis à fornecida pelo método PSPI-SS completo (operador PSPI-SS tanto para os tiros como para os receptores), com um desempenho computacional superior ao obtido com o método PSPI-SS completo.

Palavras-chave: Migração Híbrida, Migração pré-empilhamento, Migração em Duas Etapas mais Interpolação.

\footnotetext{
${ }^{1}$ Centro de Pesquisa em Geofísica e Geologia (CPGG), Universidade Federal da Bahia, 123, Campus Universitário da Federação - 40170-290 Salvador, BA, Brasil. Tel: 71-2038531; Fax: 71-2038501 - E-mail: gcap@cpgg.ufba.br

2Depto. de Geofísica Nuclear, Instituto de Física/CPGG, Universidade Federal da Bahia, 123, Campus Universitário da Federação - 40170-290 Salvador, BA, Brasil. Tel: 71-2038521; Fax: 71-2038501 - E-mail: reynam@cpgg.ufba.br
} 


\section{INTRODUCÃ̃o}

Os métodos de migração do tipo Kirchhoff (MK) (Schneider, 1978) são muito usados devido a sua eficiência computacional e flexibilidade para migrar dados sísmicos de regiões bem distintas do modelo de subsuperfície. Entretanto, na maioria das vezes esses métodos não funcionam em dados sísmicos provenientes de estruturas geológicas complexas, pois eles não incluem os tempos dos vários caminhos ("multipathing traveltimes"), ou seja, os tempos correspondentes aos multicaminhos percorridos pela energia sísmica. Esse problema é bastante mencionado na literatura geofísica e, mais recentemente, também foi discutido por Aldunate \& Pestana (2004). Os resultados dos testes utilizando-se diferentes métodos para o cálculo dos tempos de trânsito das primeiras chegadas, em modelos de extrema complexidade geológica, com a presença de corpos de sal, comprovaram uma maior dificuldade dos métodos MK em imagear corretamente os dados provenientes desses meios complexos. Por outro lado, os MK são computacionalmente eficientes e, além disso, não apresentam qualquer limitação quanto ao mergulho dos eventos, conseguindo imagear corretamente estruturas que apresentam refletores com fortes inclinações. Em meios mais simples, aqueles que apresentam estruturas quasi-plano-estratificadas, os MK funcionam a contento e conseguem reconstituir precisamente em profundidade todos os refletores.

Tomando proveito das qualidades dos MK, Tanis (1998), Aldunate e Pestana (2002) implementaram métodos híbridos de migração, aplicados a dados de famílias de tiro comum. Nesses métodos, cada ponto do modelo em profundidade é imageado de acordo com o tempo de chegada da onda direta. Ou seja, primeiro calcula-se as tabelas de tempo para cada fonte e em seguida se faz a continuação em profundidade dos dados organizados em famílias de tiro comum, através de extrapoladores em profundidade, baseados na solução da equação unidirecional da onda. E assim, em cada passo de extrapolação em profundidade, esses dois campos são cross-correlacionados e obtém-se a seção sísmica final, ou seja, a seção sísmica migrada em profundidade.

Neste trabalho, utilizamos três métodos para 0 cálculo dos tempos de trânsito das primeiras chegadas das fontes sísmicas. 0 primeiro, chamado de TGRID (Faria \& Stoffa, 1994), que é baseado na solução da equação iconal por diferenças finitas. 0 segundo, que denominamos de FAST (Stoffa, 2004), usa uma abordagem puramente geométrica para a determinação dos tempos e, portanto, obtém de forma mais rápida os tempos de chegada. 0 terceiro método, PARAX, usa o programa rayt2d pertencente ao pacote livre Seismic Unix - SU/CWP (2005), que utiliza traçados de raios com aproximação paraxial em zonas com pouco contraste de velocidade e a solução por diferenças finitas em zonas de sombra.

Os tempos de cada fonte sísmica são associados a um campo de ondas, para cada freqüência, do tipo $e^{-i \omega t(x, z)}$, que passaremos a identificar por $\mathbf{P}^{\mathbf{S}}$. E os dados sísmicos registrados são depropagados usando-se o operador de continuação "Phase Shift Plus Interpolation Split-Step" (PSPI-SS) - $W^{P S P I-S S}$ (Aldunate et al., 2004), que é uma versão mais eficiente do operador Split-Step (Stoffa et al., 1990). A extrapolação desses campos de ondas é realizada utilizando-se várias velocidades de referência, seguido de uma interpolação linear dos vários campos extrapolados em profundidade. Os dados de entrada transformados, referentes aos receptores, serão representados por $R(x, z=0, \omega)$ e sua extrapolação em profundidade, com 0 operador de extrapolação $W^{P S P I-S S}$, será indicado $\mathbf{P}^{\mathbf{R}}$.

No processo de extrapolação dos dados sísmicos, usando métodos híbridos, como aqui proposto, são utilizados dois campos de velocidades. Para realizar a extrapolação para baixo dos dados ao longo dos receptores, com o operador $W^{P S P I-S S}$, usamos um campo de velocidades em profundidade sem nenhuma suavização, pois este tipo de operador é mais sensível às pequenas variações de velocidade, de forma que quanto mais correto for o campo de velocidades, melhor será seu desempenho no processo de extrapolação. Entretanto, no cálculo dos tempos de trânsito dos campos de ondas associados às fontes, $\mathbf{P}^{\mathbf{S}}$, usamos um campo de velocidades suavizado, pois os tempos das primeiras chegadas são calculados a partir dos métodos citados acima. Portanto, como esses métodos são baseados na teoria assintótica do raio, exigem que os campos de velocidades sejam suavizados.

Os campos extrapolados em profundidade são, em cada passo do processo, cross-correlacionados e, assim, obtém-se a seção sísmica final. Ou seja, através da aplicação da condição de imagem de tempo de excitação (Chang \& McMechan, 1986). Desta forma, os refletores serão reconstituídos quando esses dois campos, referentes às fontes e aos receptores, estiverem em fase em cada ponto do modelo em profundidade (Claerbout, 1985; Yilmaz, 2001).

Para verificar a aplicabilidade desses métodos híbridos de migração pré-empilhamento, com extrapolação em duas etapas mais interpolação, implementado no domínio da frequêencia, utilizamos dois conjuntos de dados sísmicos. 0 dado sintético Marmousi e uma linha sísmica real marítima do campo "Mississippi Canyon", no Golfo do México. Os resultados obtidos com os métodos híbridos de migração apresentam imagens de boa qualidade e comparáveis com 0 resultado forne- 
cido pelo método de migração de tiro comum PSPI-SS completo (Aldunate et al., 2004).

\section{MÉTODO HÍBRIDO PSPI SPLIT-STEP}

0 método de migração pré-empilhamento no domínio da freqüência caracteriza-se por correlacionar dois campos de ondas, os campos de ondas associados às fontes e aos receptores. Os campos de ondas correspondentes às fontes, para cada freqüência $(\omega)$, neste método, é expresso por:

$$
P^{S}(x, z ; \omega)=e^{-i \omega t(x, z)},
$$

onde os tempos são determinados para todos os pontos da maIha do campo de velocidades. Os tempos de trânsito $t(x, z)$, para cada posição de fonte, são calculados a partir da solução da equação iconal com os métodos TGRID, FAST e PARAX, mencionados anteriormente.

Os campos de onda correspondentes aos receptores são continuados para baixo com 0 operador PSPI-SS (Aldunate et al., 2004):

$$
\begin{gathered}
W^{p s p i-s s}(x, z+\Delta z ; \omega) \\
=\sum_{n=1}^{N^{r e f}} \zeta_{n} \cdot e^{i p_{1}^{n} \Delta z} \cdot F_{x}^{-1} \cdot e^{i p_{2}^{n} \Delta z} \cdot F_{x},
\end{gathered}
$$

onde $N^{\text {ref }}$ é o número de velocidades de referência usados em cada passo de extrapolação em profundidade (z). $\zeta_{n}$ representa os pesos a serem usados no processo de interpolação. Os deslocamentos de fase são $p_{1}=\omega \delta u(x, z)$ e $p_{2}=k_{z}$ (onde $\left.k_{z}=\sqrt{\bar{u}^{2}(z) \omega^{2}-k_{x}^{2}}\right)$, que trabalham nos domínios $(x, \omega)$ e $(k, \omega)$, respectivamente. 0 termo de perturbação, definido a partir da vagarosidade ao invés da velocidade, é definido como $\delta u(x, z)=1 / \delta v(x, z) . F_{x}$ e $F_{x}^{-1}$ representam, respectivamente, as transformadas direta e inversa de Fourier na direção $x$. Assim, primeiro se faz a extrapolação através do deslocamento de fase com $p_{2}$, que é uma primeira correção baseada na média da vagarosidade, $\bar{u}(z)$, depois muda-se de domínio através de uma transformada espacial inversa de Fourier, para em seguida aplicar 0 segundo deslocamento de fase, $p_{1}$, que visa corrigir a variação lateral de velocidade.

No processo de extrapolação com o operador PSPI-SS, equação (2), exige-se um campo de velocidades preciso, de forma que quanto mais correto for o campo de velocidades melhor será o desempenho desse operador. Por outro lado, o cálculo dos tempos de trânsito do campo de ondas associado às fontes, $\mathbf{P}^{\mathbf{S}}$, requer um campo de velocidades suavizado, que possibilitará uma melhor estabilidade no cálculo dos tempos das primeiras chegadas.

0 esquema da migração híbrida é mostrada na Figura 1, onde exemplificamos como os dois campos de ondas (fonte e receptor) são utilizados no processo de migração. À direita, temos o campo de velocidades original, usado na extrapolação dos receptores, e à esquerda o campo de velocidades suavizado, utilizado no cálculo dos tempos de chegada da fonte sísmica em cada ponto do modelo.

Os parâmetros de suavização do campo de velocidades, tanto vertical como horizontal, foram estimados a partir do melhor resultado apresentado pela migração Kirchhoff do pacote SU/CWP (2005), por ser este tipo de migração extremamente rápida. Analisando-se a qualidade das imagens obtidas, com os vários campos suavizados, foi possível determinar o nível de suavização mais adequado para o campo de velocidades, ou seja, aquele que fornecesse uma boa imagem de subsuperfície, preservando os principais refletores do modelo geológico.

Visando simplificar a identificação dos métodos implementados, adotaremos a seguinte notação:

TGRID-MK: migração Kirchhoff com as tabelas de tempo calculadas a partir do método TGRID.

FAST-MK: migração Kirchhoff com as tabelas de tempo calculadas com o método FAST.

PARAX-MK: migração Kirchhoff com as tabelas de tempo obtidas através do método PARAX.

TGRID-PSPI-SS: método híbrido de migração usando as tabelas de tempo fornecidas pelo método TGRID e operador PSPI-SS para os receptores.

FAST-PSPI-SS: método híbrido de migração usando as tabelas de tempo calculadas com método FAST e operador PSPI-SS para os receptores.

PARAX-PSPI-SS: método híbrido de migração usando as tabelas de tempo do método PARAX e operador PSPI-SS para os receptores.

PSPI-SS completo: método de migração usando o operador PSPI-SS para as fontes e os receptores.

\section{Condição de Imagem de Tempo de Excitação}

A condição de imagem usada na migração de dados préempilhamento foi inicialmente estabelecida por Claerbout (1985), depois generalizada por Chang \& McMechan (1986), que também 


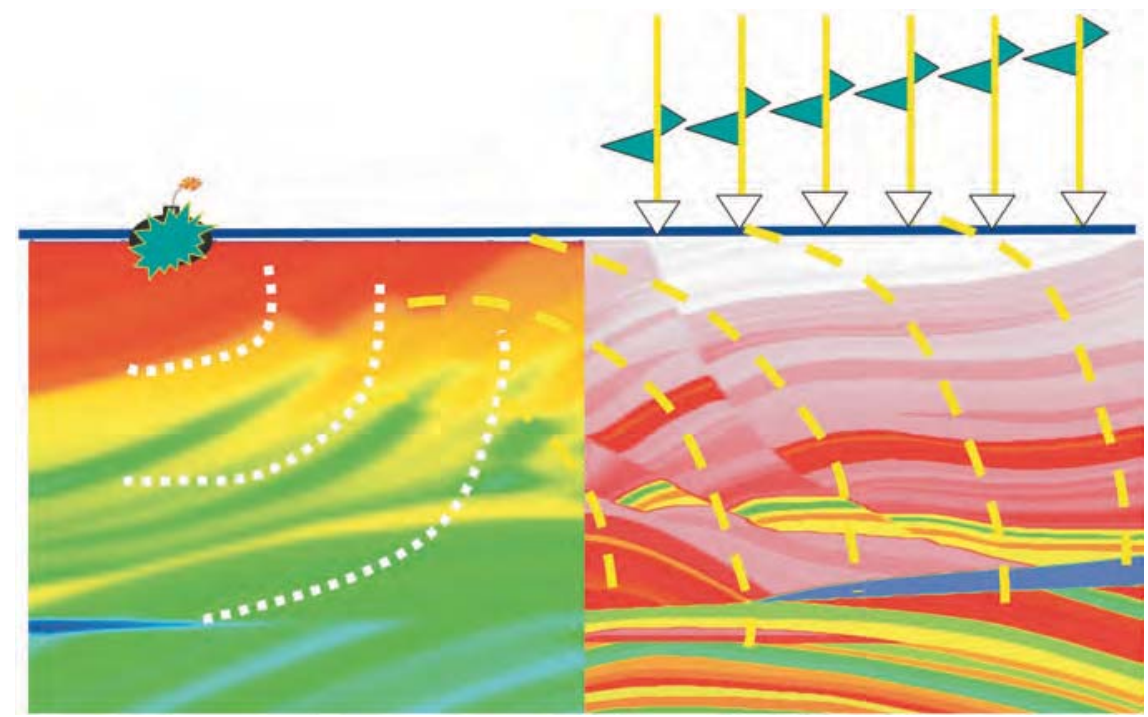

Figura 1 - Esquema mostrando a implementação do método de migração híbrido: À esquerda as isócronas de tempos de trânsito calculadas sobre um campo de velocidades suavizado e à direita o campo de onda extrapolado com o operador PSPI-SS com o campo de velocidades original. A cross-correlação no "lag" zero destes dois campos de ondas é calculada para obter a imagem migrada final.

aplicaram essa mesma condição de imagem na migração préempilhamento reversa no tempo. Ela também foi empregada na migração pré-empilhamento espectral (Tanis, 1998; Aldunate, 2002). Através dessa condição de imagem, cada ponto do modelo de subsuperfície é imageado de acordo com o tempo de chegada da onda direta ao referido ponto em profundidade. Assim, o método híbrido de migração é implementado calculando-se as tabelas de tempo para cada fonte, a partir de um campo de velocidades suavizado, em seguida é feita a extrapolação em profundidade dos dados organizados em famílias de tiro comum, através da aplicação do operador PSPI-SS, usando-se no caso de dados sintéticos, como o aqui usado para efeito de teste dos algoritmos, o modelo de velocidade original.

Assim, a condição de imagem, descrita acima, é aplicada durante o processo de continuação para baixo dos dados dos receptores (através do operador PSPI-SS), em cada passo de extrapolação em profundidade. Ou seja, os dois campos de ondas extrapolados são cross-correlacionados e a imagem final migrada em profundidade é obtida a partir da seguinte expressão:

$$
\mathbf{I}=\sum_{\omega} \mathbf{P}^{\mathbf{S}}(\omega) \cdot \mathbf{P}^{\mathbf{R}}(\omega),
$$

ou explicitando os respectivos campos de ondas, temos então:

$$
\begin{gathered}
I(x, z)= \\
\sum_{\omega} e^{-i \omega t(x, z)} \cdot W^{P S P I-S S} \cdot R(x, z=0, \omega) .
\end{gathered}
$$

Através dessa operação, os refletores são reconstituídos nas posições onde o campo de onda descendente (referente à fonte) é coincidente em tempo com o campo de onda depropagado (relativo aos receptores), em cada nível de profundidade. A imagem migrada final é obtida somando-se todas as seções de tiro comum migradas.

\section{RESULTADOS NUMÉRICOS \\ Dados Sintéticos: Modelo Marmousi}

Os dados sintéticos Marmousi é um conjunto de dados acústicos 2D, modelados a partir de uma estrutura complexa, baseado na geologia da bacia de Cuanza em Angola, convertendo-se em um teste bastante popular de algoritmos de migração. 0 modelo estrutural é dominado por falhas de crescimento, as quais se levantam desde um truncamento de sal até chegar à complicada estrutura de velocidade na parte superior do modelo. Ele tem 240 famílias de tiros, com um espaçamento entre tiros de $25 \mathrm{~m}$. Os sismogramas de tiro comum começam na posição inicial igual $3000 \mathrm{~m}$ e indo até $9000 \mathrm{~m}$. Dentro de cada família de tiro tem-se 96 traços, com intervalo de $25 \mathrm{~m}$. 0 traço de afastamento mais próximo é de $200 \mathrm{~m}$ e 0 de afastamento mais distante de $2750 \mathrm{~m}$. Cada traço sísmico possui 750 amostras a 4 ms de intervalo e, assim, cada traço contém $3 \mathrm{~s}$ de dados registrados. Dois campos de velocidades (o modelo original e sua versão suavizada) com 369 amostras (x) por 375 amostras (z), foram usados como entrada 
para o processo de migração. A Figura 2 mostra as isócronas de tempo correspondentes aos tempos $0,2,0,4,0,6,0,8,1,0, \mathrm{e} 1,2 \mathrm{~s}$, calculadas com os métodos TGRID (linha vermelha), FAST (azul) e PARAX (verde), tendo como fundo o modelo de velocidade suavizado. As diferenças entre as curvas até a isócrona de 0,6 s são pequenas, sendo as respostas dos três métodos até esse tempo considerados equivalentes, porém a partir das curvas de $0,8 \mathrm{~s}$ observa-se que cada método mostra sua própria assinatura de isócrona, que serão evidenciadas na acuracidade dos resultados finais das migrações com cada um desses métodos.

Nas Figuras 3a, 3c e 3e (coluna à esquerda) estão apresentados os resultados das migrações Kirchhoff, com os tempos da fonte calculados a partir dos métodos TGRID, FAST, PARAX, respectivamente. Na coluna à direita, mostra-se os resultados das migrações híbridas, usando as tabelas de tempo fornecidas por esses mesmos métodos de cálculo de tempo e na mesma ordem (Figuras 3b, 3d e 3f). Desses resultados, observa-se que todas as seções migradas com os métodos híbridos mostram uma notável melhoria na definição e continuidade dos refletores. Além disso, nota-se, especialmente, uma melhoria no resultado da seção migrada com 0 método TGRID-PSPI-SS (Figura $3 b)$, usando-se 3 velocidades de referência, em cada nível de profundidade, em comparação com o método TGRID-MK (Figura 3a). A diferença entre esses resultados fica ainda mais evidente na zona do anticlinal e nas falhas, onde agora é possível identificar claramente todos os eventos, sem a presença de artefatos de migração. Também uma melhor continuidade dos refletores e, portanto, uma boa focalização em toda a seção pode ser observada. Outro resultado surpreendente foi obtido com 0 método FAST-PSPI-SS (Figura 3d), se compararmos ao resultado da migração FAST-MK (Figura 3c), onde se pode observar, claramente, uma maior coerência e continuidade do anticlinal e, além disso, uma diminuição sensível dos ruídos de migração (Figura 3d), ou seja, os sorrisos de migração, na zona do anticlinal, foram diminuídos. 0 resultado obtido com a migração Kirchhoff com o método PARAX-MK (Figura 3e) é também de boa qualidade, mesmo com a migração Kirchhoff usando apenas as primeiras chegadas, que exigiu o uso de um campo de velocidades suavizado. Por outro lado, esse resultado é superado, em qualidade, pelo apresentado pelo método híbrido PARAXPSPI-SS, que apresenta uma seção migrada de altíssima qualidade e comparável aos resultados da migração PSPI-SS completo (Aldunate et al., 2004). Portanto, esse resultado (Figura 3f) é excelente para a migração dos dados Marmousi, e por isso garante que os métodos híbridos possam ser usados em dados reais sem perda de qualidade no resultado da migração.

\section{Dados Reais: Dados do Golfo do México}

Os dados marítimos do campo de petróleo "offshore" do "Mississippi Canyon", localizado no Golfo do México, foram adquiridos em uma região que se caracteriza pela presença de um corpo de sal gigante e achatado e por estratificações horizontais ao seu redor, especialmente embaixo desse corpo de sal. A Figura 4 ilustra 0 modelo de velocidades usado nas migrações.

A linha sísmica marítima 2D processada contém 1001 tiros, espaçados de 26,67 m, onde cada família de tiro é formada por 180 receptores, também espaçados de 26,67 m. Cada traço tem $6,0 \mathrm{~s}$ de registro, amostrados a cada $4 \mathrm{~ms}$, ou seja, um total de 1501 amostras por traço sísmico. 0 afastamento mais próximo é de 100,58 m, e a linha sísmica foi adquirida na disposição "endon" - tiro na frente. A malha do campo de velocidades possui 1000 pontos na direção horizontal $(x)$ por 1185 na vertical (z), com intervalo de amostragem horizontal de 26,67 m e 6,67 m na vertical.

Para o cálculo das tabelas de tempo usou-se uma versão suavizada do campo de velocidades (Figura 4), com os parâmetros de suavização estimados de forma análoga como foi feito para os dados Marmousi. A Figura 5 mostra as curvas de isócronas, nos tempos $0,7,1,1,1,4,1,9$ e $2,7 \mathrm{~s}$. As curvas na cor vermelha correspondem ao resultado obtido com o método TGRID, as linhas em azul com método FAST e as linhas na cor verde com o método PARAX. Até $1,1 \mathrm{~s}$ as curvas isócrinas apresentam um comportamento bastante similar, entretanto, diferenciando-se muito a partir da isócrina de $1,4 \mathrm{~s}$, e mostrando que esses métodos apresentam limitações no cálculo dos tempos de trânsito em regiões salinas.

Para efeito de comparação temos na Figura 6 o resultado obtido com a migração PARAX-MK, que apresenta principalmente uma boa delineação do corpo de sal e também dos refletores embaixo do mesmo. Mas como na região que delineia o corpo de sal (ver detalhe do corpo de sal - Figura 7) existe uma variação lateral forte de velocidade, associada com um pequeno mergulho, podemos notar ainda a presença de artefatos embaixo dessa região. Por outro lado, o tempo gasto para migrar, apenas um tiro, foi de aproximadamente $45 \mathrm{~s}$, em um computador com processador Pentium IV de 2,8 GHz e 2 GB de RAM. Portanto, apresentando um excelente desempenho computacional que é uma das grandes virtudes dos métodos Kirchhoff.

Os resultados das migrações com os métodos híbridos TGRID-PSPI-SS, FAST-PSPI-SS e PARAX-PSPI-SS são mostrados nas Figuras 8a, 8b e 8c, respectivamente. Verificamos nesses resultados uma excelente delineação da base rugosa do corpo de sal e também uma boa continuidade dos refletores, diferenciando- 


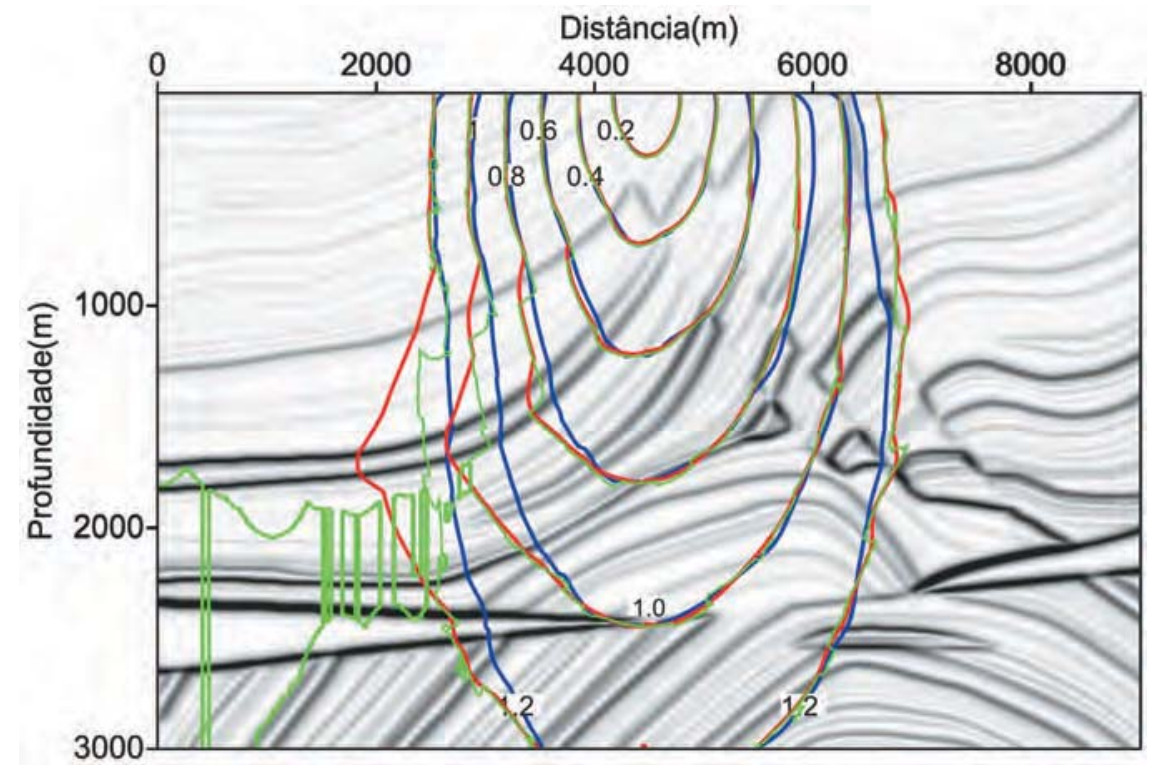

Figura 2 - Curvas isócronas obtidas a partir do modelo de velocidades Marmousi suavizado, correspondentes aos tempos 0,2, 0,4, 0,6, 0,8, 1,0 e 1,2 s, com os métodos TGRID (linha vermelha), FAST (linha azul) e PARAX (linha verde).

se somente nos detalhes (Figuras 9c, 9d e 9e). A Figura 9 apresenta um detalhe da parte do corpo de sal, onde temos a função pseudo-refletividade na Figura 9a e nas demais figuras os resultados das migrações testadas.

Comparando-se os resultados citados, o método PARAXPSPI-SS foi o que apresentou o melhor resultado desses métodos híbridos, pois mostra uma melhor delineação da base do corpo de sal, associado com uma diminuição de ruídos nesta parte da imagem migrada. 0 tempo requerido para a migração de um tiro comum, com cada um dos métodos híbridos mencionados, usandose apenas três velocidades de referência, foi aproximadamente de 2 min.

Agora, se compararmos o resultado do método PARAX-PSPISS (Figura 8c) com o resultado do método PSPI-SS completo (Figura 7), notamos que as diferenças são apenas de detalhes, sendo ambas de mesma qualidade. No detalhe do corpo de sal, com esse último método, Figura 9f, pode-se observar uma pequena melhoria no refletor que delineia a base do sal, e também uma boa diminuição dos artefatos de migração. Já o tempo de migração de cada tiro comum com o método PSPI-SS completo, usando-se três velocidades de referência, foi de $5 \mathrm{~min}$.

\section{CONCLUSÕES}

Neste trabalho verificamos a aplicabilidade e o desempenho dos métodos híbridos de migração pré-empilhamento em profundi- dade TGRID-PSPI-SS, FAST-PSPI-SS e PARAX-PSPI-SS, em dados organizados em famílias de tiro comum, tanto em dados sintéticos, como em dados reais de alta complexidade estrutural. Para o cálculo dos tempos de trânsito das fontes, foram empregados os métodos TGRID, FAST e PARAX, que associados a um campo de ondas $\mathbf{P}^{\mathbf{S}}$ empregado na condição de imagem de tempo de excitação, visando aumentar a eficiência computacional dos métodos espectrais de migração.

Os resultados das migrações obtidos com os dados sintéticos Marmousi e os tempos de trânsito, calculados a partir dos métodos TGRID, FAST e PARAX, mostraram-se adequados e superando em qualidade ao resultado apresentado pelo método Kirchhoff. Especialmente, os métodos de migração PARAXPSPI-SS e FAST-PSPI-SS apresentaram excelentes resultados, comparáveis aos da migração PSPI-SS completo. E assim, comprovando a robustez desses métodos híbridos de migração, mesmo em dados sísmicos provenientes de estruturas geológicas complexas.

Os resultados numéricos obtidos com os dados reais do Golfo, serviram para aferir que os métodos híbridos podem fornecer imagens migradas de melhor qualidade, quando comparadas às imagens conseguidas com as dos métodos de migração Kirchhoff. Verificando-se que nesse dado o corpo de sal e os eventos embaixo de fortes contrastes de velocidades ficaram bem imageados pelos métodos híbridos. Além disso, o custo computacional pôde ser reduzido em até 50\%, em relação ao exigido 


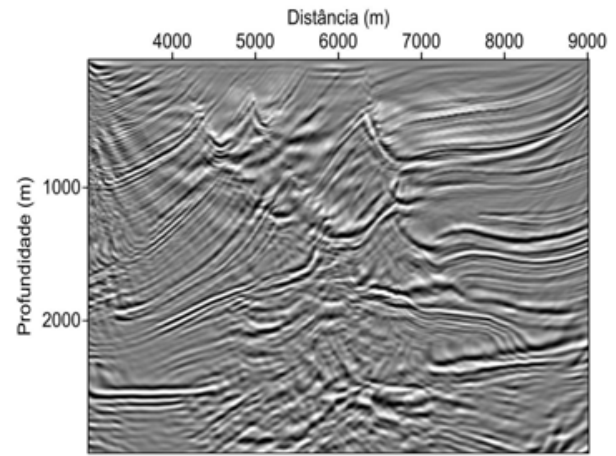

(a)

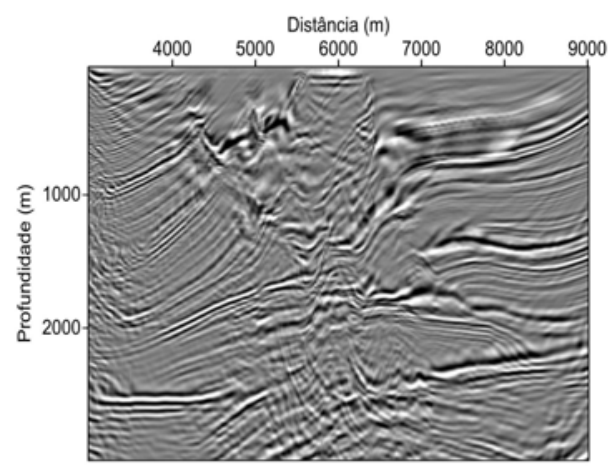

(c)

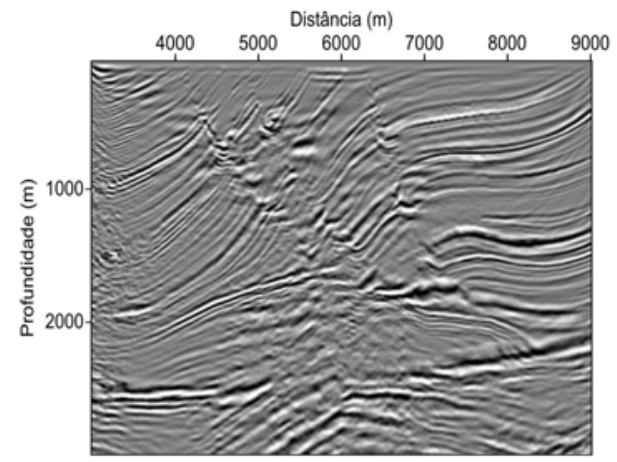

(e)

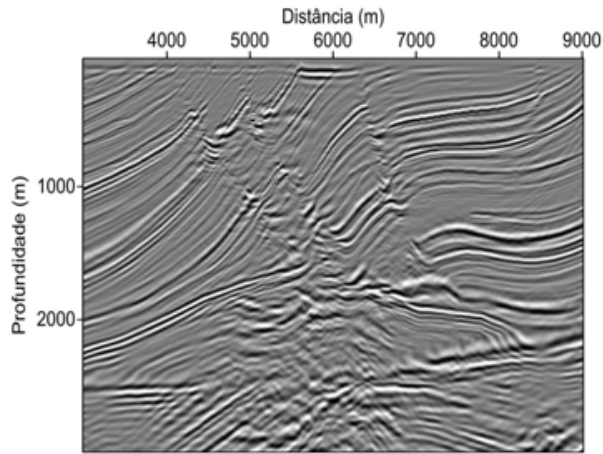

(b)

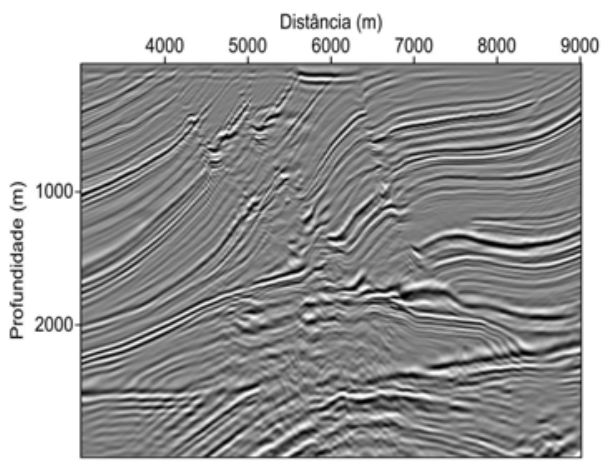

(d)

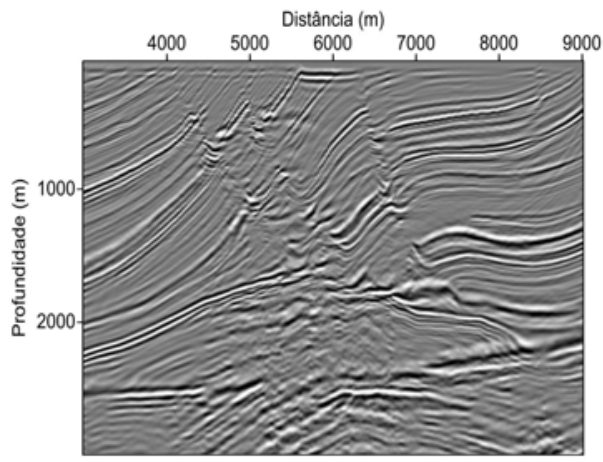

(f)

Figura 3 - Resultados das migrações dos dados Marmousi com os métodos híbridos: Resultados com os métodos TGRID-MK (a), TGRID-PSPI-SS (b), FAST-MK (c), FAST-PSPI-SS (d), PARAX-MK (e) e PARAX-PSPI-SS (f), respectivamente. Os melhores resultados são os mostrados nas Figuras (d) e (f).

pelo método de migração PSPI-SS completo.

Ressaltamos ainda que o desempenho desses métodos híbridos depende diretamente da acurácia do operador de extrapolação e também do grau da precisão empregado no cálculo dos tempos de trânsito. Ou seja, poderemos obter resultados de qualidade cada vez melhor à medida que os tempos de trânsito sejam mais precisos e os operadores de extrapolação mais corretos.

\section{AGRADECIMENTOS}

Os autores agradecem ao CTPETRO/CNPq-FINEP pelo apoio à rede Cooperativa de Pesquisa em Risco Exploratório, à Agência Nacional de Petróleo (ANP), PETROBRAS e ao CPGG/UFBA pelo apoio no desenvolvimento deste trabalho. Reynam Pestana agradece ao CNPq pelo suporte, através de bolsa de pesquisa, processo 300.312/88-1 (RM). E também aos revisores da RBGf deste artigo pelos valiosos comentários e sugestões. Agradecemos 


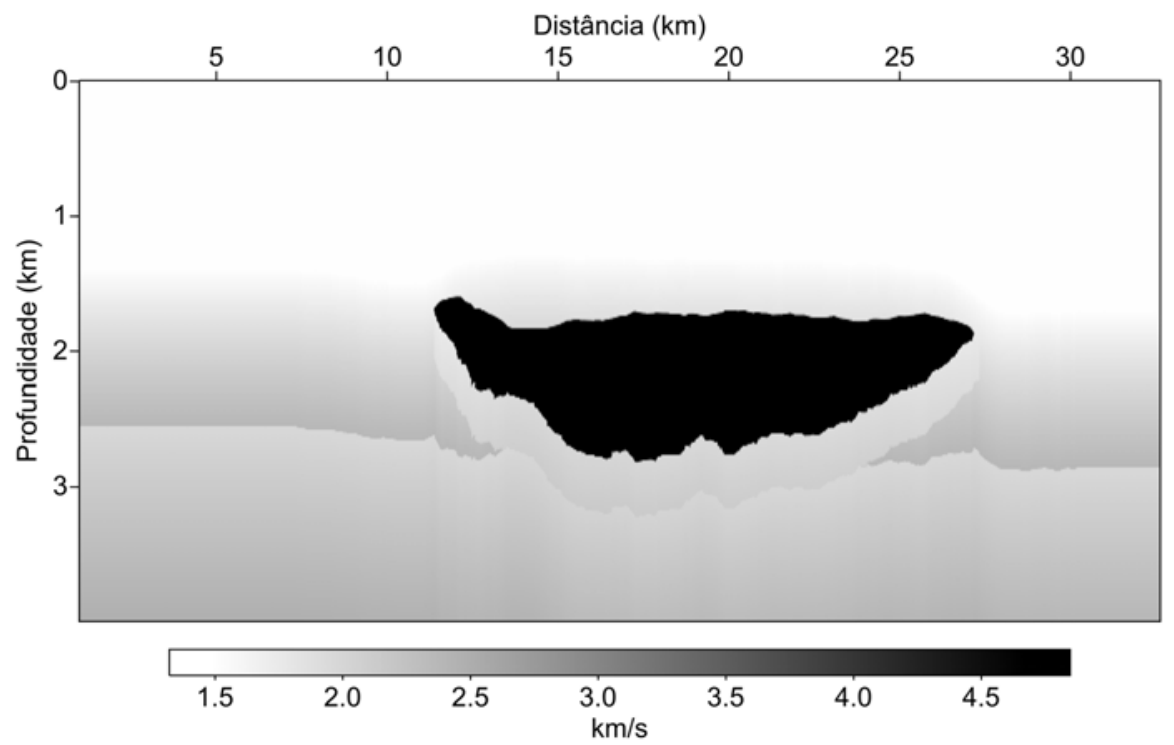

Figura 4 - Campo de velocidades do Golfo do México usado para a migração.

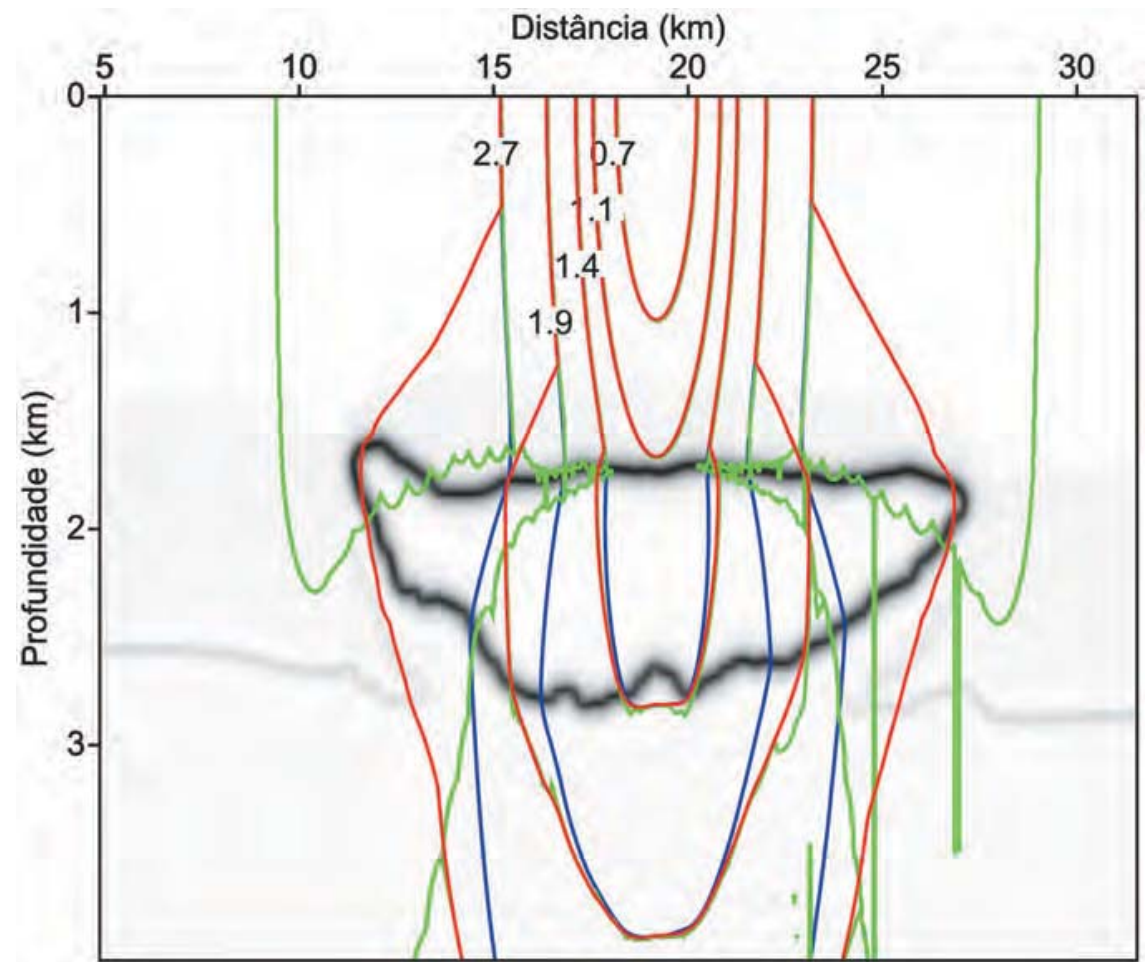

Figura $\mathbf{5}$ - Isócronas mostrando o resultado dos métodos de cálculo de tempo de trânsito na presença de um corpo de sal. São mostradas as curvas dos tempos 0,7,1,1,1,4,1,9, 2,7 s, com o método TGRID (linha vermelha), método FAST (linha azul) e o método PARAX (linha verde). Ao fundo uma versão suavizada do campo de velocidades do Golfo do México usada para o cálculo dos tempos. 


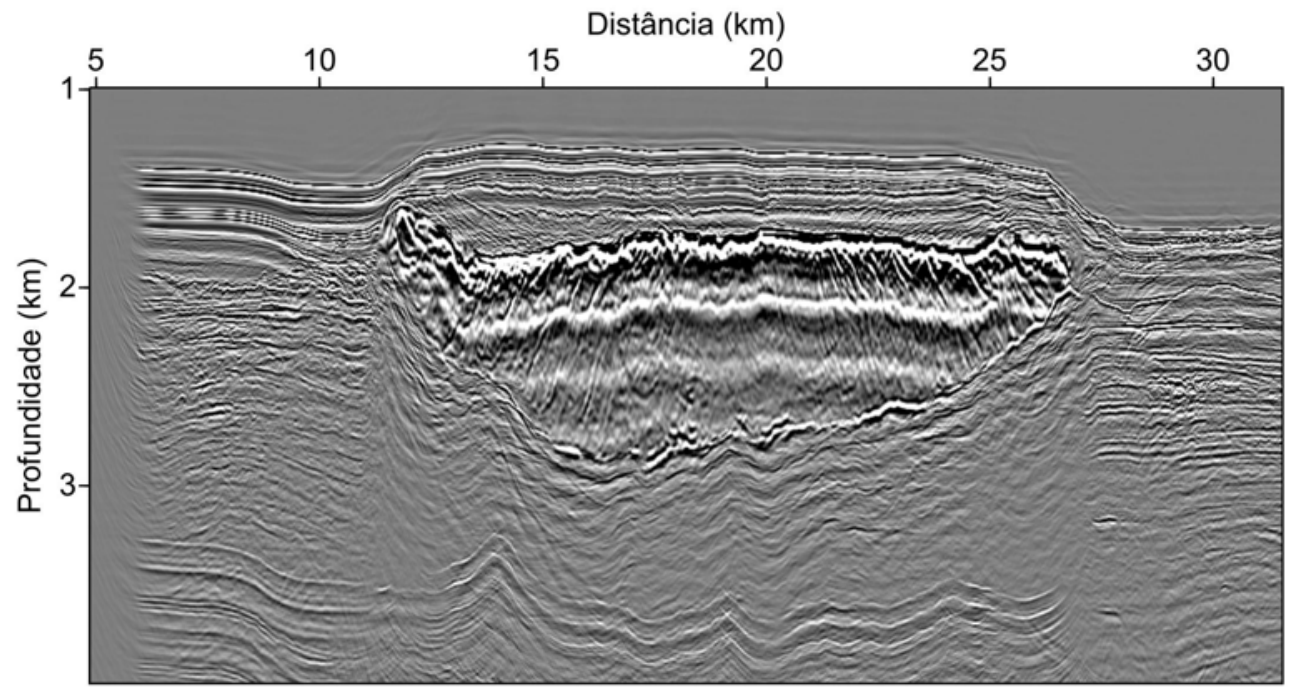

Figura 6 - Resultado da migração Kirchhoff do pacote SU dos dados do Golfo do México, com os tempos calculados pelo método PARAX.

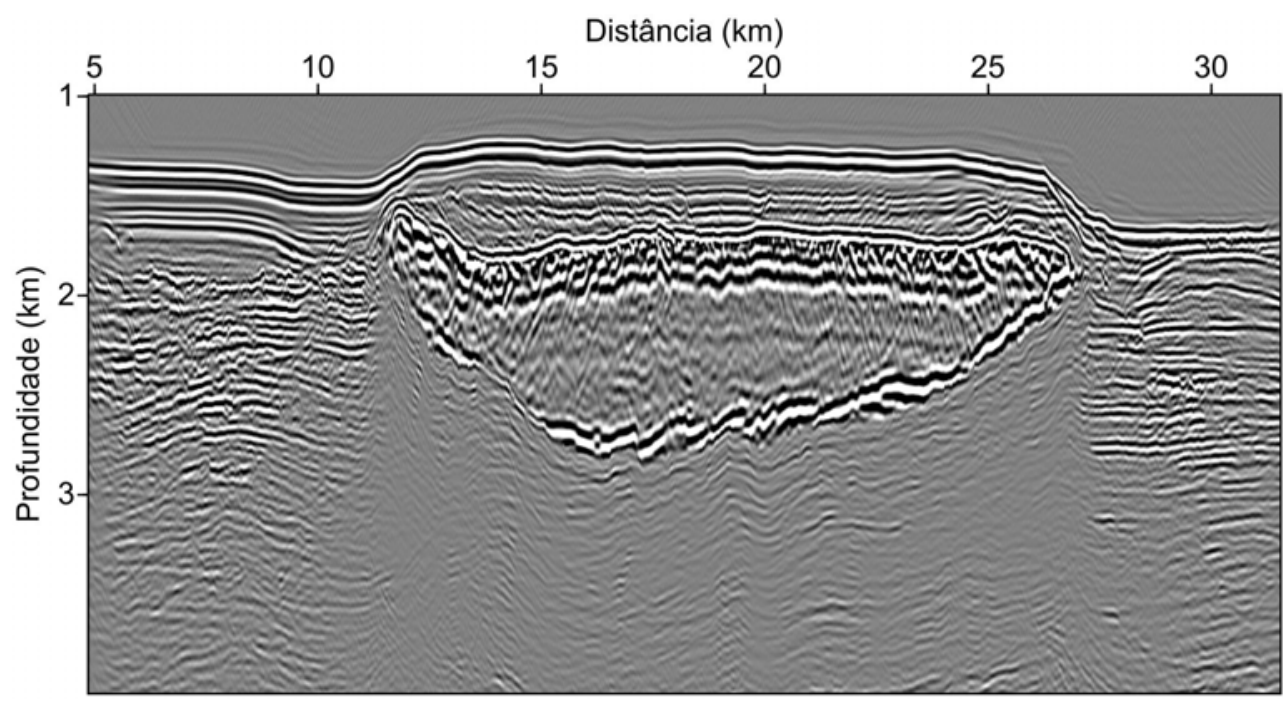

Figura 7 - Seção migrada com o método PSPI-SS completo.

ainda a Antoinne Guiton do Stanford Exploration Project (SEP) pelo fornecimento do campo de velocidades dos dados do Golfo do México.

\section{REFERÊNCIAS}

ALDUNATE GC. 2002. Migração pré-empilhamento usando operadores de extrapolação tipo "split-step", Dissertação de mestrado, Universidade Federal da Bahia.

ALDUNATE GC \& PESTANA RC. 2004. Imageamento de estruturas geológicas associadas a fortes mergulhos com métodos de migração MK e WEM. In: Congresso Brasileiro de Geologia, 42, 2004, Araxá. Anais eletrônico... Araxá: SBG, 2004. CD-ROM.

ALDUNATE GC, PESTANA RC \& STOFFA PL. 2004. Migração sísmica 2D pré-empilhamento em profundidade com operadores de extrapolação "split-step", Revista Brasileira de Geofísica, 22: 153-161.

CHANG WF \& McMECHAN GA. 1986. Reverse-time migration of offset vertical seismic profiling data using the excitation-time imaging condition, Geophysics, 51: 67-84.

CLAERBOUT JF. 1985. Imaging the Earth's Interior: Blackwell Scientific.

FARIA EL \& STOFFA PL. 1994. Traveltime computation in Transversely isotropic media, Geophysics, 59: 272-281. 


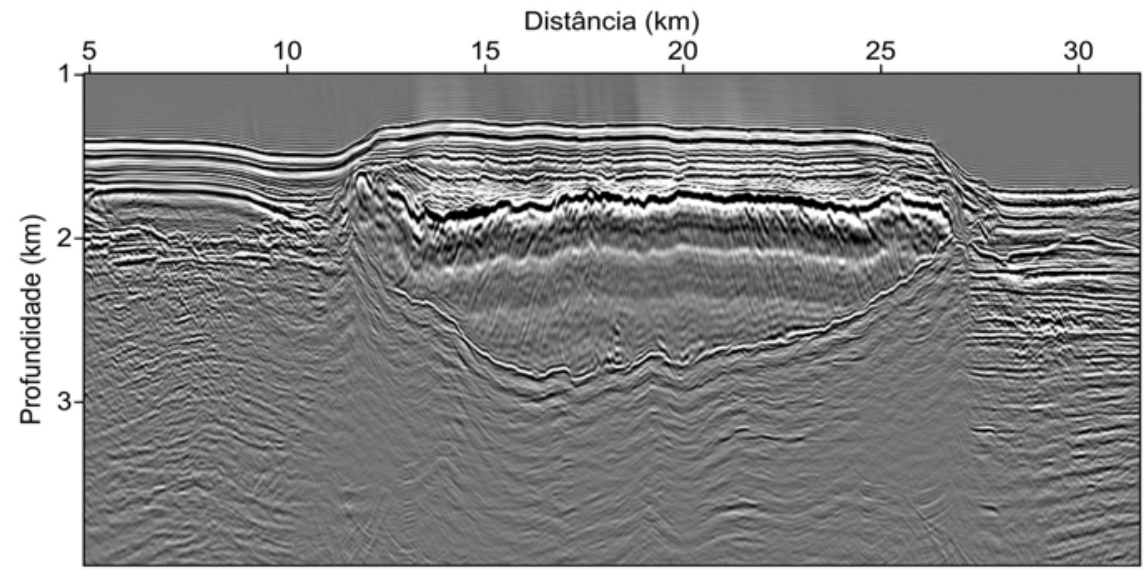

(a)

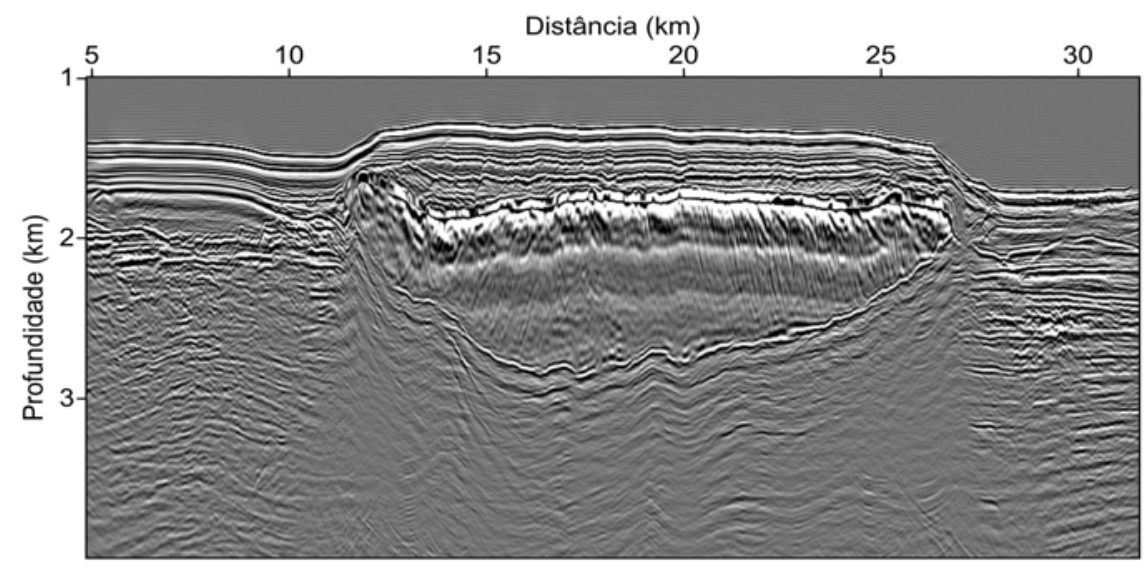

(b)

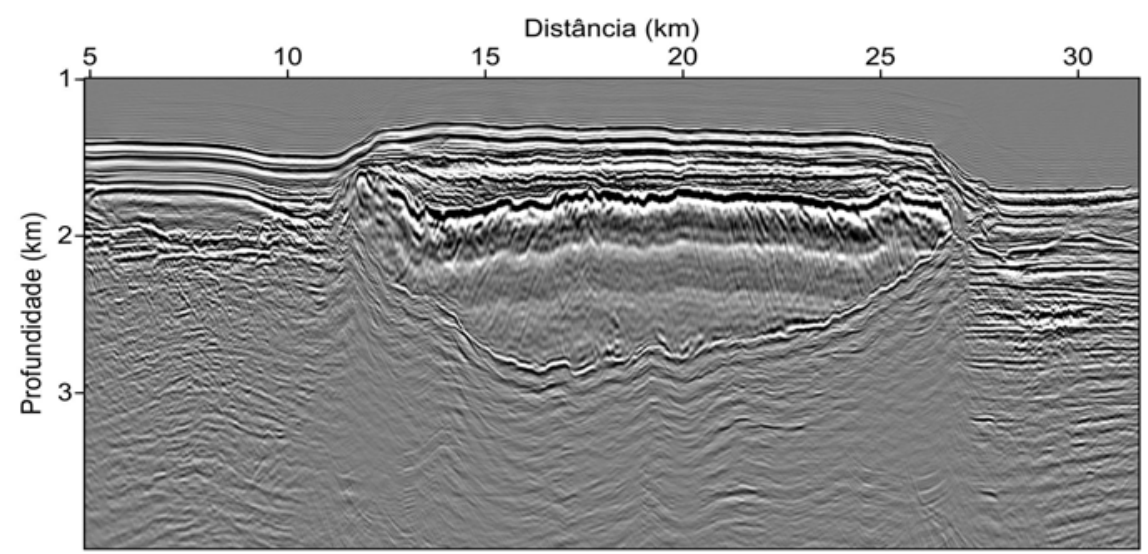

(c)

Figura 8 - Resultados das migrações dos dados do Golfo do México com os métodos híbridos: TGRID-PSPI-SS (a), FAST-PSPI-SS (b) e PARAX-PSPI-SS (c). Comparando-se os resultados com o mostrado na Figura 7, concluímos que os resultados fornecidos pelos métodos híbridos são muito parecidos com o obtido pelo método PSPI-SS completo. 


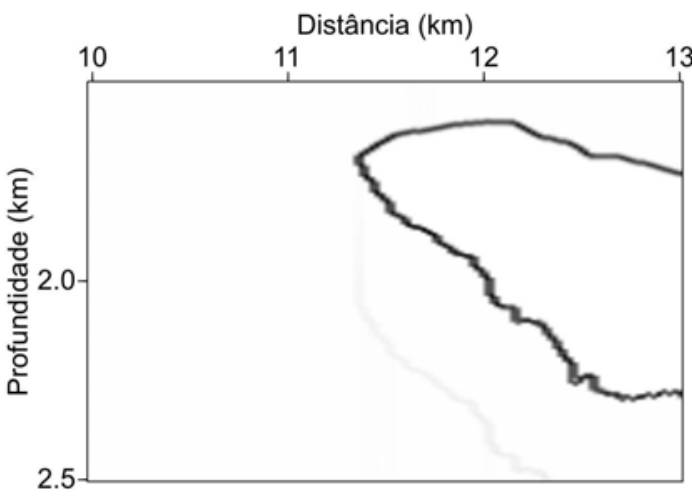

(a)

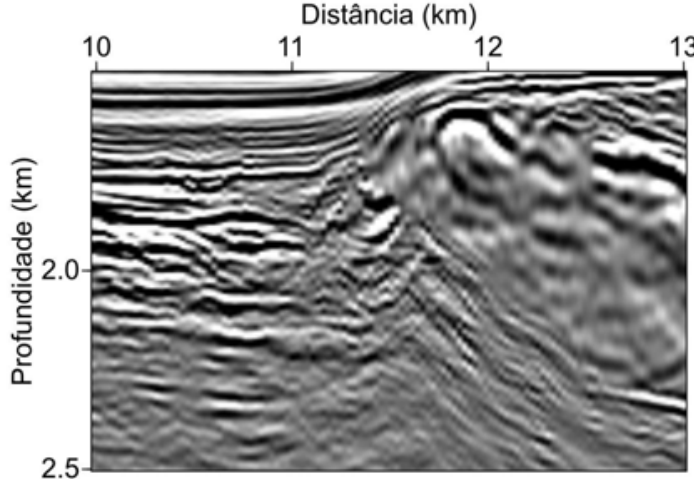

(c)

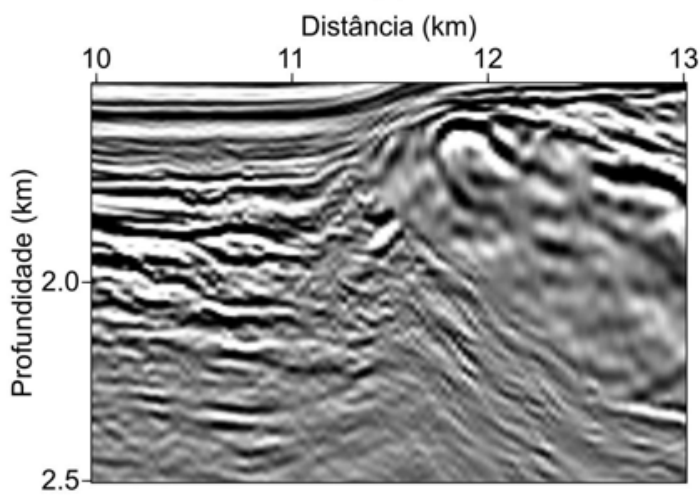

(e)

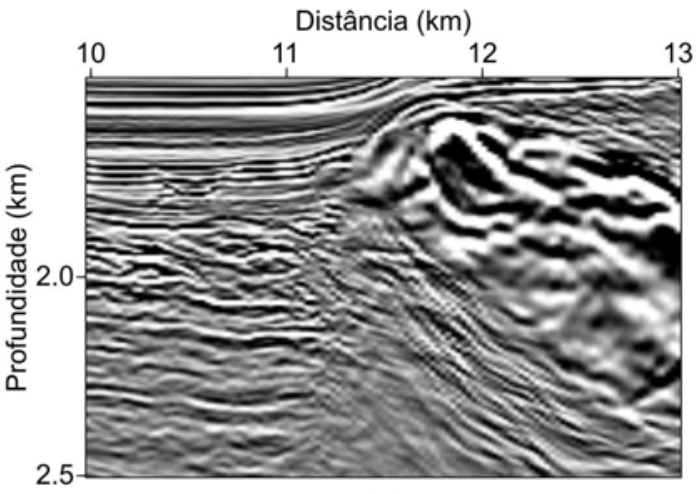

(b)

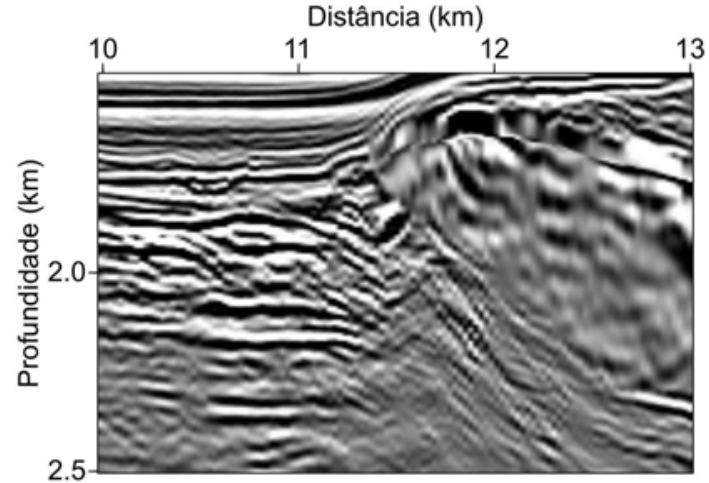

(d)

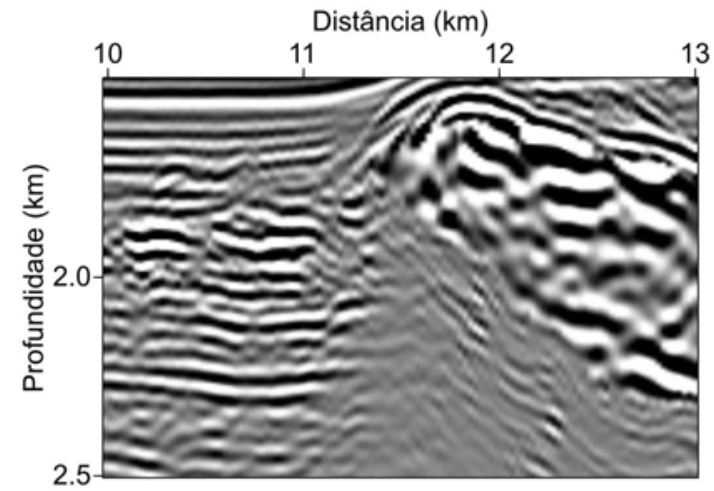

(f)

Figura 9 - Detalhe de uma parte do corpo de sal usada para comparar o delineamento do corpo de sal apresentado pelos resultados das migrações. A Pseudo-refletividade (a) mostra que existe um alto contraste de velocidade ao redor do corpo de sal. Resultado do método PARAX-MK (b), TGRIDPSPI-SS (c), FAST-PSPI-SS (d), PARARX-PSPI-SS (e) e PSPI-SS completo (f).

SCHNEIDER WA. 1978. Integral formulation for migration in two and three dimensions, Geophysics, 43: 49-76.

STOFFA PL. 2004. Programa de cálculo de tempos (FAST), Comunicação Pessoal.

STOFFA PL, FOKKEMA JT, FREIRE RML \& KESSINGER WP. 1990. Splitstep migration, Geophysics, 55: 410-421.

SU-CWP. 2005. Pacote de Processamento Sísmico - Seismic Unix (SU),
Center Wave Phenomena, versão 38, Colorado School of Mines

TANIS CM. 1998. Prestack split-step fourier depth migration algorithms and parallel implementations on Cray T3E, Ph.D Thesis in geophysics, University of Texas.

YILMAZ 0. 2001. Seismic data analysis, Society of Exploration Geophysicists, Tulsa. $2027 \mathrm{p}$. 


\section{NOTAS SOBRE OS AUTORES}

Gary C. Aldunate é graduado em Física (UATF-B0/1997), mestre em Geofísica de Exploração de Petróleo (UFBA/2002) e atualmente é aluno do curso de Doutorado em Geofísica da Universidade Federal da Bahia. As suas principais áreas de interesse são modelagem e processamento de dados sísmicos com ênfase em migração de dados sísmicos.

Reynam C. Pestana é graduado em Física (UFBA/1983) e doutor em Geofísica pela UFBA, 1988. Pós-doutorado em Geofísica no Instituto de Geofísica da Universidade de Karlsruhe (Alemanha), 1989-1991, e de 1998-1999 no Instituto de Geofísica da Universidade do Texas em Austin. De 1988 até 0 presente é pesquisador do CPGG/UFBA, atuando no Grupo de Geofísica de Exploração de Petróleo. Desde 1992 é Professor Adjunto do Departamento de Geofísica Nuclear do Instituto de Física e da Pós-graduação em Geofísica da UFBA. Tem atuado no desenvolvimento de métodos e algoritmos de processamento e imageamento sísmicos. Mais recentemente suas pesquisas envolvem técnicas de migração de dados sísmicos decompostos em ondas planas. É membro da SBGf e da SEG. 